\title{
Mitochondrial Protective Role of Curcumin in Sodium Valproate- Induced Nephrotoxicity in Male Rats
}

\author{
MOEIN SHANEH ${ }^{1}$ \\ ${ }^{1}$ University of Medical Sciences, Mazandaran, Iran MA, Department of Toxicology, Faculty of Pharmacy, Mazandaran
}

\begin{abstract}
Sodium valproate, a medicine for epilepsy and other neuropsychiatric disorders, damages kidney tissue, especially its mitochondria. Thus, the current research was aimed at evaluating the potential of curcumin against the sodium valproate-induced nephrotoxicity. Animals utilized in this research were categorized into six groups including the first group as control, the second as valproate $(500 \mathrm{mg} / \mathrm{kg})$, the third, fourth, and fifth groups as rats treated with a combination of valproate and curcumin ( 25 up to $100 \mathrm{mg} / \mathrm{kg}$ ), and the sixth group only was administrated with curcumin $(100 \mathrm{mg} / \mathrm{kg})$. All the treatments were performed for six consecutive weeks. The results indicated that the function and swelling significantly increased while membrane potential decreased in kidney mitochondria by using $100 \mathrm{mg} \mathrm{kg}-1$ curcumin. Moreover, the protein carbonyl and malondialdehyde significantly decreased in kidney tissue, whereas glutathione slightly increased after curcumin treatment. Taken together, our findings for the first time suggested that curcumin confers mitochondrial protection against sodium valproate-induced nephrotoxicity and can be used to antagonize sodium valproate nephrotoxicity.
\end{abstract}

Keywords: Curcumin; Kidney; Mitochondria; Nephrotoxicity.

\section{INTRODUCTION}

Valproate, and its valproic acid, sodium valproate (SVP), and valproate semisodium forms, are primarily utilized for the epilepsy therapy and other neuropsychiatric onditions (Oztopuz et al. 2020). As observed, the SVP can cause serious renal damage as proximal renal tubule dysfunction and interstitial nephritis (Gad 2018). Based on the previous reports, reactive oxygen species (ROS) is the main mediator of SVP-induced toxicity, where ROS production has been recorded in terms of teratogenicity, hepatotoxicity, renal toxicity, and also in neurotoxicity (Said and El-Agamy 2010).

Curcumin is a biologically active compound that can be found in the rhizomes of Curcuma longa $L$ (Uzunisarcikli and Aslanturk 2019). This bright yellow chemical is utilized widely as herbal medicine, food preservative, and spice in a variety of countries worldwide (Strimpakos and Sharma 2008). Curcumin is a phenolic ingredient with a broadspectrum of biological functions, like antioxidant and antimutagenic, antineoplastic, and anti-inflammatory (Yang et al. 2020). To date, many studies have focused on the curcumin properties and its effect on the antioxidant system, etc. For instance, Ahmida et al. (2012) observed that curcumin ameliorates the vancomycin-induced decline in the content of glutathione and the activity of antioxidant enzymes.

Aslanturk and Uzunhisarcikli (2020) showed that daily treatment with $100 \mathrm{mg} / \mathrm{kg}$ curcumin alleviates the histological alterations, the activity of antioxidant enzyme, and the level of lipid peroxidation brought about by BPA chemical. The antioxidant activity of this phenolic compound is more potent several times than vitamin $\mathrm{E}$, which is a powerful antioxidant that assists repair damaged cells and possesses a function for protecting the kidney against the peroxidation of lipids in aminoglycoside antibiotics (Farombi and Ekor 2006; Ahmida 2012).

However, the exact mechanism underlying the mitochondrial protective effect of curcumin on the kidney is unclear. Thus, the current research was aimed to examine the mitochondrial protective efficacy of curcumin treatment against sodium valproate-induced nephrotoxicity in male rats.

\section{MATERIAL AND METHODS}

Animals and Study Design: Male rats weighing 20-30 g were obtained from the Research Center of Laboratory Animals, Mazandaran University of Medical Sciences, Sari, Iran. Animals were maintained under standard conditions of $12 \mathrm{~h}$ light cycle, temperature $23 \pm 2{ }^{\circ} \mathrm{C}$, with easy access to water and food. All experimental methods were performed based on the ethical protocols and standards approved by the Animal Experimentation Committee.

A total of 30 male rats were categorized randomly into six groups. During the treatment period, the rats were injected intraperitoneally one time daily (at 12:00). The groups were treated according to the following schedule: Group 1 received normal saline (control); Group 2 was provided with VPA along [500 $\mathrm{mg} / \mathrm{kg}$ ] with the vehicle; Group 3 was provided with VPA + curcumin [25 mg/kg]; Group 4 was provided with VPA + curcumin [50 mg/kg]; Group 5 was provided with VPA + curcumin [100 mg/kg]; Group 6 was provided with curcumin along [100 mg/kg] with the vehicle. All the treatments were performed for six consecutive weeks.

\section{Mitochondrial parameters:}

Pathological imaging: Animals were sacrificed under anesthesia one day after the last injection. The kidney tissue was immediately isolated from other tissues and removed as described by Heidari et al. (2018). The samples were fixed in $10 \%$ formaldehyde solution, dehydrated in alcohols, and embedded in paraffin. The kidney tissue was stained with hematoxylin-eosin (H\&E) stain and evaluated by using Nikon Labophot light microscope as elucidated by Aslanturk and Uzunisarcikli (2020).

Isolation of the kidney mitochondria: Isolation of kidney mitochondria was carried out as elucidated by Heidari et al. (2018). Briefly, rat kidneys were homogenized in isolation buffer (2 mM HEPES, 0.1\% bovine serum albumin, $70 \mathrm{mM}$ sucrose, $0.5 \mathrm{mM}$ EGTA, and $220 \mathrm{mM}$ mannitol, $\mathrm{pH}=7.4$ ) at 
a 10:1 buffer to kidney ratio ( $\mathrm{v}$ : w) by using an IKA homogenizer $(7000 \mathrm{rpm}, 15 \mathrm{sec})$. The homogenates were then centrifuged at $1,500 \times \mathrm{g}$ for $30 \mathrm{~min}$ at $4^{\circ} \mathrm{C}$ to discard intact cells and nuclei. The supernatants were further centrifuged at $10,000 \times \mathrm{g}$ for $15 \mathrm{~min}$ at $4^{\circ} \mathrm{C}$ for precipitating the mitochondria. All steps for isolating of kidney mitochondria were carried out on the ice or at $4^{\circ} \mathrm{C}$ for minimizing mitochondrial injuries.

Mitochondrial function: Mitochondrial function was evaluated via tracking the reduction of 3-[4,5dimethylthiazol-2-yl]-2,5-diphenyltetrazolium bromide (MTT) as a colorimetric procedure as elucidated by GhaziKhansari et al. (2006).

Mitochondrial swelling: Mitochondrial swelling was measured according to light scattering shifts, which tacked by the spectrophotometric tool at $540 \mathrm{~nm}$ as elucidated by Zhao et al. (2010). The absorbance was recorded at 540 $\mathrm{nm}$ by using an ELISA reader (Tecan, Rainbow Thermo, Austria) was utilized for determining. The reduced absorbance is considered as a parameter of enhanced mitochondrial swelling.

Mitochondrial membrane potential (MMP): To determine the kidney MMP, the mitochondrial uptake of the cationic fluorescent dye Rhodamine 123 was utilized. In this procedure, $10 \mu \mathrm{M}$ of this dye was added to the kidney mitochondria in buffer MMP (Taghizadeh et al. 2020). The fluorescence spectrophotometer V-1600 PC (, Shimadzu, Japan) (emission: $535 \mathrm{~nm}$ and excitation: $490 \mathrm{~nm}$ ) was used to measure the fluorescence.

Lipid peroxidation: Malondialdehyde (MDA) as a parameter of membrane lipid peroxidation (Shahani et al. 2016) was determined according to Zhang et al. (2008). Herein, 0.25 $\mathrm{ml}$ phosphoric acid $(0.05 \mathrm{M})$ was combined with $0.2 \mathrm{ml}$ of homogenate along with $0.3 \mathrm{ml}$ of $0.2 \%$ TBA. All specimens were then transferred to a hot-water bath for 35 minutes, and then to an ice bag for $5 \mathrm{~min}$. Then, $0.5 \mathrm{ml}$ of $\mathrm{n}$-butanol was combined with each tube and centrifuged at 3000rpm for $15 \mathrm{~min}$. The MDA level of each specimen was determined by an ELISA reader (Tecan, Rainbow Thermo, Austria) at $532 \mathrm{~nm}$.

Glutathione content: To estimate the glutathione (GSH) content, $0.1 \mathrm{ml}$ of tissue combined with the $0.1 \mathrm{~mol} / \mathrm{l}$ of phosphate buffer and $0.04 \%$ Ellman's reagent in a total volume of $3.0 \mathrm{ml}, \mathrm{PH} 7.4$. The yellow color intensity was recorded by using $\mathrm{V}-1600 \mathrm{PC}$ spectrophotometer (Shimadzu, Japan) at $412 \mathrm{~nm}$. The content of glutathione was presented as nM (Naserzadeh et al. 2016).

Protein carbonyl concentration: To determine the protein carbonyl content as a parameter of protein oxidative damage (Jamshidzadeh et al. 2017), $200 \mu \mathrm{l}$ of tissue was homogenated in $500 \mu \mathrm{l}$ of $20 \%$ trichloroacetic acid (w/v). The precipitate was exposed to $500 \mu$ l of $0.2 \% 2,4$ dinitrophenylhydrazine and $500 \mu \mathrm{l}$ of $2 \mathrm{~N} \mathrm{HCl}$ for the control group. Each sample was centrifuged and washed three times intermittently with $1 \mathrm{ml}$ of the ethanol-ethyl acetate and eventually dissolved in $200 \mu \mathrm{l}$ of $6 \mathrm{M} \mathrm{GuHCl}$. The protein carbonyl concentration was estimated via recording the absorbance at $365 \mathrm{~nm}$ wavelengths (Koohsari et al. 2016).

Statistical analysis: Data in the current study are presented as mean $\pm \mathrm{SD}$. A comparison of data sets was carried out via the one-way analysis of variance along with the post hoc Tukey's mul-tiple test. A value of $\mathrm{P}<0.05$ was assumed significant.

\section{RESULTS}

The microscopic evaluation of the kidney tissue of the sodium valproate-treated animals demonstrated that valproate resulted in the falling of urinary tract epithelial cells and the expansion of the Bowman's capsule space. However, curcumin in the presence of sodium valproateinduced nephrotoxicity in male rats showed improvement in the term of kidney damages (Figure 1).

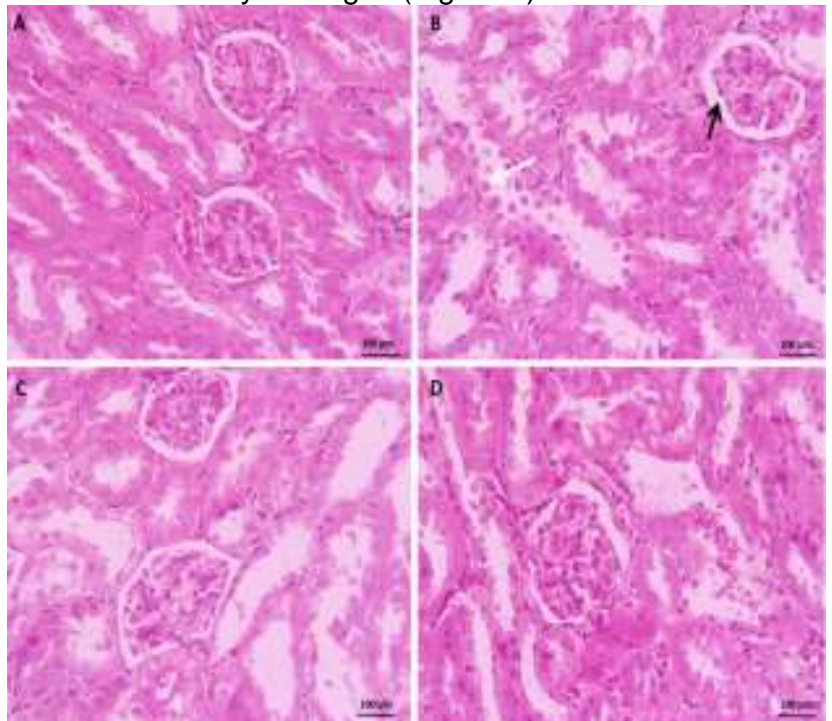

Figure 1. H\&E-stained kidney tissue of control and sodium valproate-treated rats. White arrow: Falling off urinary tract epithelial cells; Black arrow: Expansion of the Bowman's capsule space; A: Normal saline; B: Sodium valproate; C: Curcumin $(50 \mathrm{mg} / \mathrm{kg})$ + Sodium valproate; D: Curcumin $(100 \mathrm{mg} / \mathrm{kg})+$ Sodium valproate.

Efficacy of curcumin on kidney mitochondrial function: As presented in Figure 2, the use of sodium valproate resulted in a significant decrement in kidney mitochondrial function $(P<0.001)$. As compared to the sodium valproate group, the injection of curcumin improved significantly the function of kidney mitochondria $(P<0.01)$. The results indicated that at a concentration of $100 \mathrm{mg} \mathrm{kg}-1$, the curcumin was significantly more effective in attenuating the sodium valproate-induced mitochondrial damage when compared to others (Figure 2). 


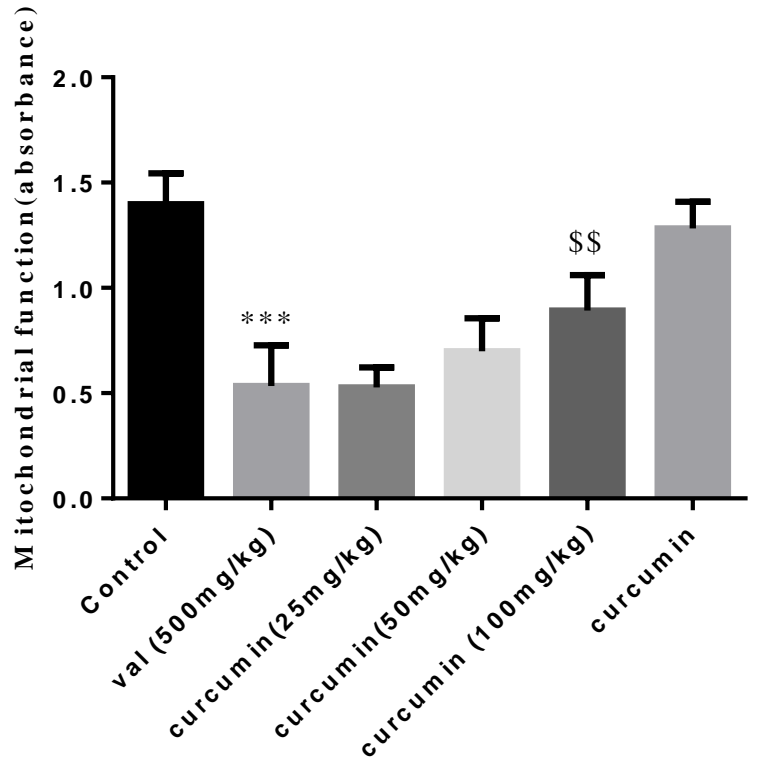

Figure 2. Mitochondrial Protective effect of administration of curcumin on the mitochondrial function in sodium valproate-treated rats. Notably, curcumin group (the last column on the right) comprised of rats that were just administrated by $100 \mathrm{mg} \mathrm{kg}-1$ curcumin. Data represent mean \pm SEM ( $n=5$ rats, per group). The ${ }^{* * *}$ show $P<0.001$ when compared to control group.

The $\$ \$$ indicate $P<0.01$ when compared to sodium valproate group. Statistical analysis was performed via one-way ANOVA followed by the post hoc Duncan's multiple-range test, respectively.

Efficacy of curcumin on kidney mitochondrial swelling: As demonstrated in Figure 3 , the administration of sodium valproate resulted in a significant decrement in kidney mitochondrial swelling $(P<0.001)$. However, the use of curcumin at high concentration (100 mg kg-1) significantly increased the level of swelling in kidney mitochondria $(P<0.05)$ (Figure 3).

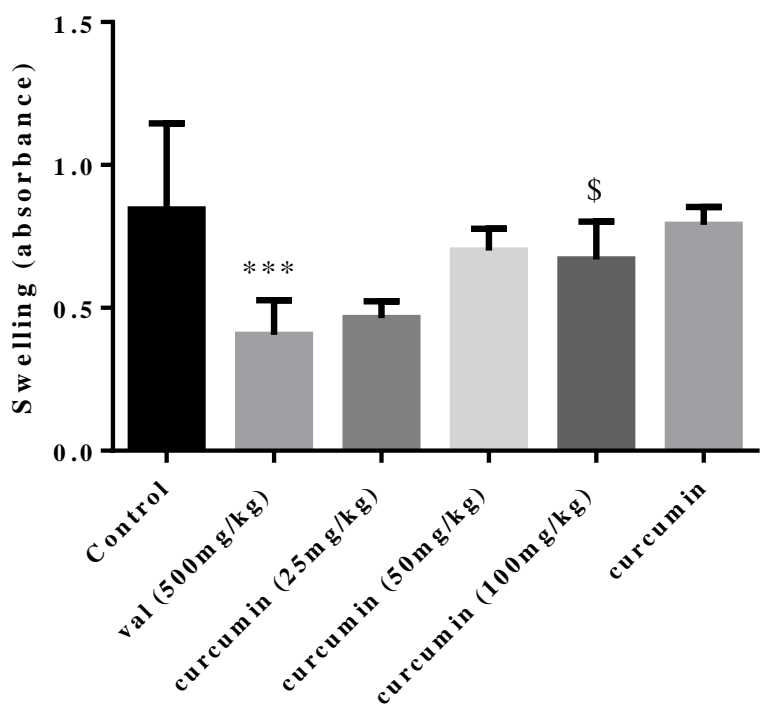

Figure 3. Mitochondrial Protective effect of administration of curcumin on the swelling in sodium valproate-treated rats. Notably, curcumin group (the last column on the right) comprised of rats that were just administrated by $100 \mathrm{mg}$ $\mathrm{kg}-1$ curcumin. Data represent mean $\pm \operatorname{SEM}$ ( $n=5$ rats, per group). The ${ }^{* * *}$ show $P<0.001$ when compared to control group. The $\$$ indicate $P<0.05$ when compared to sodium valproate group. Statistical analysis was performed via one-way ANOVA followed by the post hoc Duncan's multiple-range test, respectively.

Efficacy of curcumin on kidney mitochondrial membrane potential (MMP): The subject of kidney mitochondria to sodium valproate promoted the MMP to a significantly higher level than of that recorded in the control $(P<0.05)$. However, $100 \mathrm{mg} \mathrm{kg}-1$ curcumin significantly reduced the MMP $(P<0.01)$ (Figure 4).

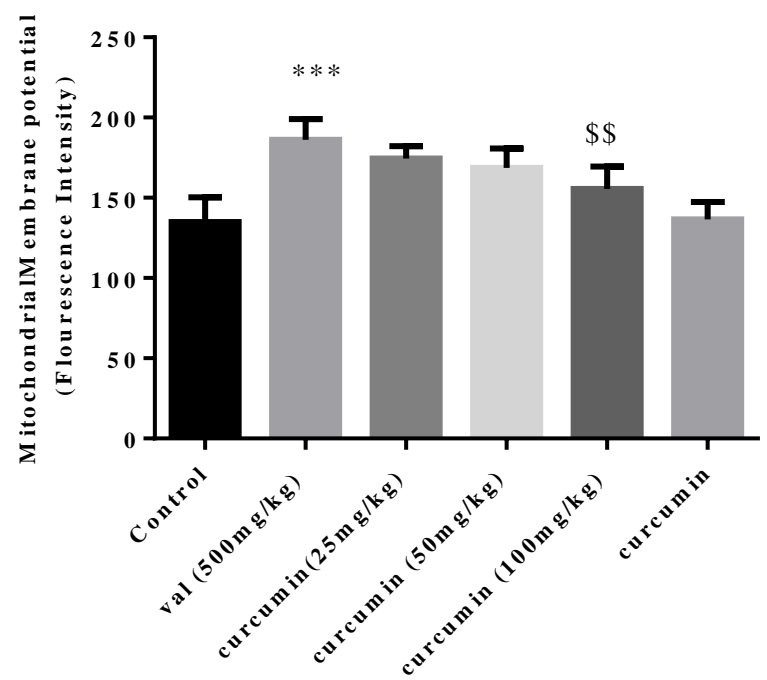

Figure 4. Mitochondrial Protective effect of administration of curcumin on the mitochondrial membrane potential in sodium valproate-treated rats. Notably, curcumin group (the last column on the right) comprised of rats that were just administrated by $100 \mathrm{mg} \mathrm{kg}-1$ curcumin.

Data represent mean \pm SEM ( $n=5$ rats, per group). The ${ }^{\star * *}$ show $P<0.001$ when compared to control group. The $\$ \$$ indicate $P<0.01$ when compared to sodium valproate group. Statistical analysis was performed via one-way ANOVA followed by the post hoc Duncan's multiple-range test, respectively

Efficacy of curcumin on kidney mitochondrial lipid peroxidation: The administration of sodium valproate to kidney mitochondria significantly increased the accumulation of MDA, as a parameter of lipid peroxidation, to a higher level than was that registered in the control $(P<$ 0.001 ). As demonstrated in Figure 5 , the exposure of kidney mitochondria to curcumin decreased the MDA to a significantly lower level than of that recorded in the sodium valproate group $(P<0.05)$. 


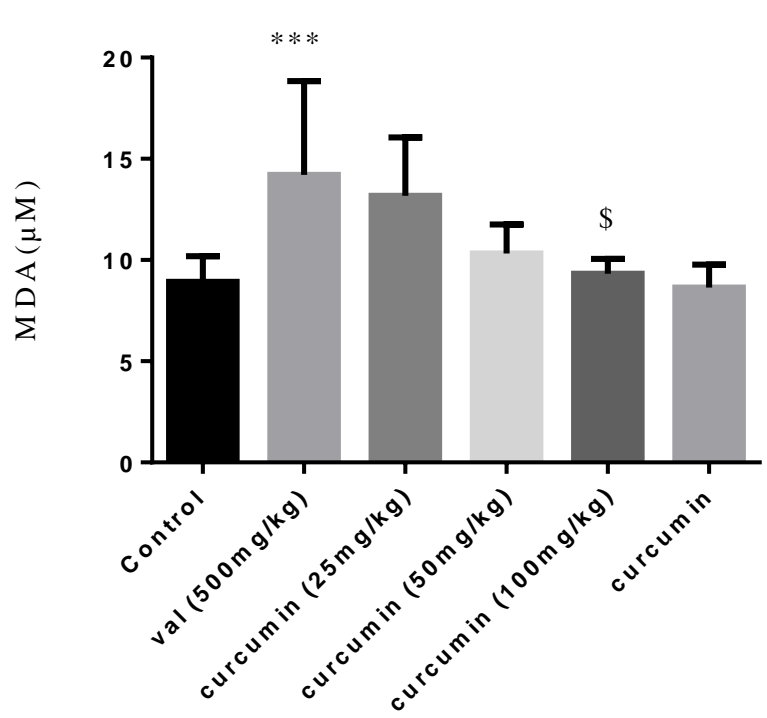

Figure 5. Mitochondrial Protective effect of administration of curcumin on the malondialdehyde (MDA) in sodium valproate-treated rats. Notably, curcumin group (the last column on the right) comprised of rats that were just administrated by $100 \mathrm{mg} \mathrm{kg}-1$ curcumin.

Data represent mean \pm SEM ( $n=5$ rats, per group). The ${ }^{* * *}$ show $P<0.001$ when compared to control group. The $\$$ indicate $P<0.05$ when compared to sodium valproate group. Statistical analysis was performed via one-way ANOVA followed by the post hoc Duncan's multiple-range test, respectively.

Efficacy of curcumin on kidney mitochondrial glutathione: The level of glutathione was found to be decreased $\left(P_{<}\right.$ 0.05 ) after the generation of reactive oxygen species in the group treated with sodium valproate. The concentration of glutathione, however, was insignificantly increased after the administration of curcumin at all concentrations (Figure 6).

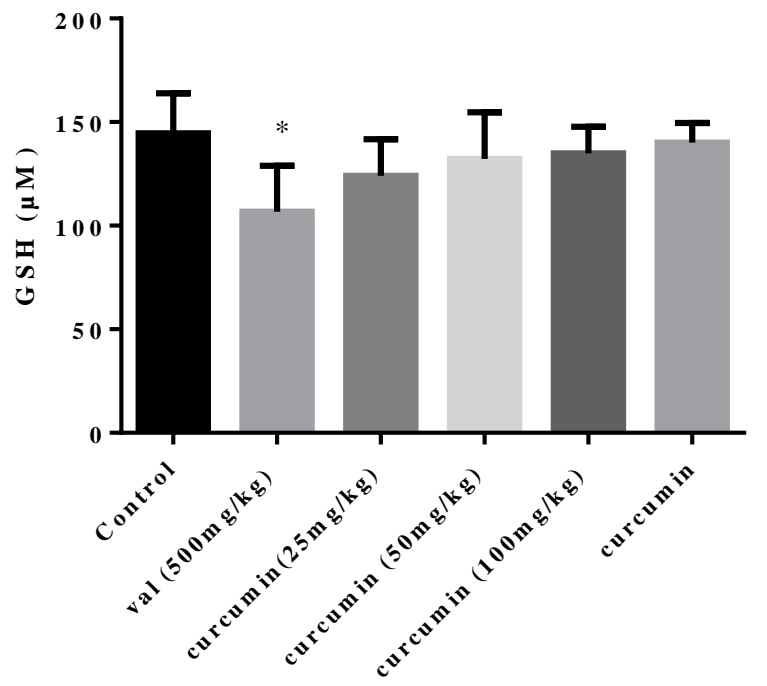

Figure 6. Mitochondrial Protective effect of administration of curcumin on the glutathione (GSH) in sodium valproatetreated rats. Notably, curcumin group (the last column on the right) comprised of rats that were just administrated by $100 \mathrm{mg} \mathrm{kg}-1$ curcumin. Data represent mean \pm SEM $(n=5$ rats, per group). The * show $P<0.05$ when compared to control group. Statistical analysis was performed via oneway ANOVA followed by the post hoc Duncan's multiplerange test, respectively.

Efficacy of curcumin on kidney mitochondrial protein carbonyl: Unlike sodium valproate, different concentrations of curcumin significantly decreased the level of protein carbonyl in mitochondria isolated from the kidney ( $p<$ 0.05). The reducing effect on the concentrations 50 and $100 \mathrm{mg} \mathrm{kg}$-1 of curcumin could decline protein carbonyl content more than $25 \mathrm{mg} \mathrm{kg}-1$ curcumin $(P<0.05)$ (Figure $7)$. In other words, curcumin declined protein carbonyl of kidney mitochondria in a concentration-dependent manner.

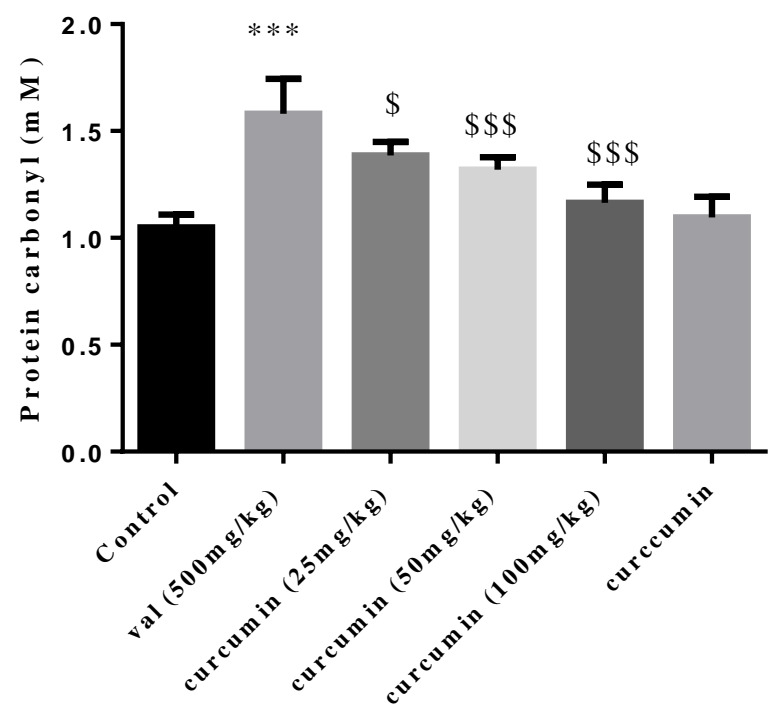

Figure 7. Mitochondrial Protective effect of administration of curcumin on the protein carbonyl in sodium valproatetreated rats. Notably, curcumin group (the last column on the right) comprised of rats that were just administrated by $100 \mathrm{mg} \mathrm{kg}-1$ curcumin. Data represent mean \pm SEM $(n=5$ rats, per group). The ${ }^{* * *}$ show $P<0.001$ when compared to control group.

The $\$, \$ \$$, and $\$ \$ \$$ indicate, $P<0.05, P<0.01$ and $P<0.001$, respectively, when compared to sodium valproate group. Statistical analysis was performed via one-way ANOVA followed by the post hoc Duncan's multiple-range test, respectively.

\section{DISCUSSION}

The current research demonstrates that daily administration of sodium valproate led to nephrotoxicity. This finding is in agreement with the reported nephrotoxic effect of sodium valproate following administration to rats (Said and El-Agamy 2010). A range of researchers has attributed sodium valproate-induced toxicity to fatty acid $\beta$ oxidation in the damaged mitochondrial (Oztopuz et al. 2010). The reactive metabolite of sodium valproate leads to depletion of glutathione, resulting in mitochondrial dysfunction (Gad 2018). As a result, oxidative stress, as an output of overproduction of oxygen radicals and attenuation 
of antioxidant capacity, has been suggested to act as a key function in the etiology of sodium valproate-induced cytotoxicity (Said and El-Agamy 2010).

Novel achievements demonstrated that sodium valproate toxicity involves oxidative stress-induced via the combination of GSH depletion and ROS generation (Oztopuz etal. 2010). Thus, any mechanism which activates GSH dependent enzymes, prevents GSH depletion, and/or decline ROS may provide protection against sodium valproate-induced nephrotoxicity.

Curcumin decreased the lipid peroxidation, in turn, alleviated the disturbance of the mitochondrial membrane and the decline of mitochondrial membrane potential, which had been induced by sodium valproate. Similarly, Aslanturk and Uzunisarcikli (2020) revealed that curcumin declines lipid peroxidation and in turn protects the mitochondrial membrane against bisphenol A-induced damages. SotoUrquieta et al. (2014) also demonstrated curcumin can restore mitochondrial function and decrease lipid peroxidation in diabetic $\mathrm{db} / \mathrm{db}$ mice kidneys. Moreover, curcumin increased the content of glutathione, as an important source for the preservation of thiol in the proteins located in the mitochondrial membrane (Oztopuz et al. 2010). Oxidation of the thiol group facilitates structural shifts happening in the pore complexes that induce the mitochondrial permeability transition (Shokrzadeh et al. 2014). The opening of pores allows infinite movements of solutes/water and mitochondria undergo conformational shifts referred to as swelling of mitochondria (Mehdizadeh et al. 2017). As observed in our study, a high concentration of curcumin enhanced mitochondrial swelling. Similarly, Ahmida (2012) revealed the protective role of curcumin in vancomycin-induced nephrotoxic oxidative damage by changing the mitochondrial function and swelling. Curcumin may ameliorate mitochondrial dysfunction via suppressing the activity of glycogen synthase kinase- $3 \beta$, which located downstream of the Akt signaling pathway (Wang et al. 2020). Overall, the mitochondrial assessments emphasize the mitochondrial protective function of curcumin in valproate-activated nephrotoxicity in rats.

The observed function of curcumin in the alleviation of valproate-induced nephrotoxicity can be elucidated by its preventive features against oxidative stress. So, this phenolic ingredient increased glutathione and significantly decreased MDA and protein carbonyl content of kidney mitochondria in a concentration-dependent manner.

These findings are in light with the observed efficacy of curcumin in alleviating of histological alterations, antioxidant enzyme activities, and lipid peroxidation level in the rat nephrotoxicity (Aslanturk and Uzunisarcikli 2020; Venkatesan et al. 2010).

Curcumin by its capability chelating metal ions, inhibiting oxidative enzymes, quenching oxygen radicals, interacting with oxidative cascade, and scavenging free radicals suppresses lipid peroxidation and eventually restores the antioxidant capacity (Kamalakkannan et al. 2005; Wei et al. 2006). This capability of curcumin is largely originated from de novo biosynthesis of glutathione. The biologically active compound can stimulate the activity of glutamate-cysteine ligase, an important rate-limiting enzyme in glutathione biosynthesis, via inducing expression of the gene coding the subunits of glutamate- cysteine ligase (Zheng et al. 2007; Reyes-Gordillo et al. 2007).

Moreover, curcumin can increase de novo biosynthesis of glutathione via raising the activity of glutathione- S-transferase, which is involved in glutathione production (Nishinaka et al. 2007).

Based on our findings, several modes of action can be explained for the observed mitochondrial protective function of curcumin in sodium valproate-induced nephrotoxicity. The first mode of action is the improvement of mitochondrial function swelling, and membrane potential, the second is the suppression of lipid/protein peroxidation, and the third is the prevention of glutathione depletion. These mechanisms potentiate kidneys to endure valproateinduced nephrotoxicity and support our hypothesis that with curcumin administration in valproate-treated rats, nephrotoxicity can be alleviated.

\section{CONCLUSION}

Taken together, our findings suggested that curcumin administration confers protection against sodium valproateinduced nephrotoxicity with regard that curcumin could produce considerable protection with no side effects on valproate-treated rates. Curcumin attenuated oxidative damage in rat kidney by declining intracellular ROS production and protecting mitochondria from oxidative stress. However, further clinical researches are required to prove the mitochondrial protective role of curcumin.

Disclosure statement: The authors report no conflicts of interest.

\section{REFERENCES}

1. Aslanturk A, Uzunhisarcikli M. 2020. Protective potential of curcumin or taurine on nephrotoxicity caused by bisphenol A. Environ Sci Pollut Res. doi:10.1007/s11356-020-08716-1

2. Ahmida MHS. 2012. Protective role of curcumin in nephrotoxic oxidative damage induced by vancomycin in rats. Exp Toxicol Pathol. 64:149-153. doi:10.1016/j.etp.2010.07.010

3. Farombi E, Ekor M. 2006. Curcumin attenuates gentamicininduced renal oxidative damage in rats. Food Chem Toxicol. 44:1443-8. doi:10.1016/j.fct.2006.05.005 Gad A M. 2018. Study on the influence of caffeic acid against sodium valproate-induced nephrotoxicity in rats. J Biochem Mol Toxicol. 32:e22175. doi:10.1002/jbt.22175

4. Ghazi-khansari M, Mohammadi-Bardbori A, Hosseini MJ. 2006. Using janus green $B$ to study paraquat toxicity in rat liver mitochondria role of ACE inhibitors (Thiol and Nonthiol ACEi). Annals New York Acad Sci. 1090: 98-107. doi:10.1196/annals.1378.010

5. Heidari R, Ahmadi A, Mohammadi H, Ommati MM, Azarpira N, Niknahad H. 2018. Mitochondrial dysfunction and oxidative stress are involved in the mechanism of methotrexate-induced renal injury and electrolytes imbalance. Biomed Pharmacother. 107:834-840. doi:10.1016/j.biopha.2018.08.050

6. Kamalakkannan N, Rukkumani R, Varma PS, Viswanathan P, Rajasekharan KN, Menon VP. 2005. Comparative effects of curcumin and an analogue of curcumin in carbon tetrachloride- induced hepatotoxicity in rats. Basic Clin Pharmacol Toxicol. 97(1):15-21. doi:10.1111/j.17427843.2005.pto_97103.x

7. Koohsari M, Shaki F, Jahani D. 2016. Protective Effects of Edaravone against Methamphetamine-Induced 
cardiotoxicity. Braz arch biol technol. 59: e16160093. doi:10.1590/1678-4324-2016160093

8. Jamshidzadeh A, Niknahad H, Heidari R, Zarei M, Ommati MM, Khodaei F. 2017. Carnosine protects brain mitochondria under hyperammonemic conditions: Relevance to hepatic encephalopathy treatment. Pharma Nutrition. 5: 58-63. doi:10.1016/j.phanu.2017.02.004

9. Mehdizadeh H, Pourahmad J, Taghizadeh G, Vousooghi N Yoonessi A, Naserzadeh P, Behzadfar L, Rouini MR, Sharifzadeh M. 2017. Mitochondrial impairments contribute to spatial learning and memory dysfunction induced by chronic tramadol administration in rat: Protective effect of physical exercise. Prog Neuropsychopharmacol Biol Psychiatry. doi:10.1016/j.pnpbp.2017.07.022

10. Naserzadeh $P$, Shariatmadari R, Gholami M, Rouini MR, Sharifzadeh M. 2016. Protective effects of physical exercise on MDMA-induced cognitive and mitochondrial impairment Free Radic Biol Med. 99: 11-19. doi:10.1016/j.freeradbiomed.2016.07.018

11. Nishinaka T, Ichijo $Y$, Ito $M$, Kimura $M$, Katsuyama M, Iwata K, Miura T, Terada T, Yabe-Nishimura C. 2007. Curcumin activates human glutathione-Stransferase P1 expression through antioxidant response element. Toxicol Lett. 170(3):238-47. doi:10.1016/j.toxlet.2007.03.011.

12. Oztopuz O, Turkon H, Buyuk B, Coskun O, Hilal Sehitoglu M, Akif Ovali M, Uzun M. 2020. Melatonin ameliorates sodium valproate-induced hepatotoxicity in rats. Mol Biol Rep. 47:317-325. doi:10.1007/s11033-019-05134-6

13. Reyes-Gordillo K, Segovia J, Shibayama M, Vergara P, Moreno MG, Muriel P. 2007. Curcumin protects against acute liver damage in the rat by inhibiting NF-kB, proinflammatory cytokines production and oxidative stress. Biochim Biophy Acta. 1770(6):989-96. doi:10.1016/j.bbagen.2007.02.004

14. Said SAA, El-Agamy DS. 2010. Prevention of sodium valproate-induced hepatotoxicity by curcumin, rosiglitazone and $\mathrm{N}$-acetylcysteine in rats. Arzneimittelforschung. 60(11):647-53. doi:10.1055/s-0031-1296342

15. Shokrzadeh M, Shaki F, Mohammadi E, Rezagholizadeh N, Ebrahimi F. 2014. Edaravone Decreases Paraquat Toxicity in A549 Cells and Lung Isolated Mitochondria. Iran J Pharm Res. 13 (2): 675-681.

16. Shahani S, Behzadfar F, Jahani D, Ghasemi M, Shaki F. 2016. Antioxidant and antiinflammatory effects of nasturtium officinale involved in attenuation of gentamicin induced nephrotoxicity. Toxicol Mech Method. doi:10.1080/15376516.2016.1258748

17. Soto-Urquieta M G, López-Briones S, Pérez-Vázquez V, Saavedra-Molina A, González-Hernández G A, RamírezEmiliano J. 2014. Curcumin restores mitochondrial functions and decreases lipid peroxidation in liver and kidneys of diabetic db/db mice. Biol Res. 47(1): 74. doi:10.1186/07176287-47-74

18. Strimpakos AS, Sharma RA. 2008. Curcumin: preventive and therapeutic properties in laboratory studies and clinical trials. Antioxid Redox Signal. 10:511-545. doi:10.1089/ars.2007.1769

19. Taghizadeh G, Mehdizadeh H, Pourahmad J. Foroumadi A, Hassani S, Halvaei Khankahdani Z, Noruzi M, Behmadi H, Lavasani H, Rouini MR, Sharifzadeh M. 2020.

20. Bucladesine Attenuates Spatial Learning and Hippocampal Mitochondrial Impairments Induced by 3, 4Methylenedioxymethamphetamine (MDMA). Neurotox Res. 38:38-49. doi:10.1007/s12640-020-00183-3

21. Uzunisarcikli M, Aslanturk A. 2019. Hepatoprotective effects of curcumin and taurine against bisphenol A-induced liver injury in rats. Environ Sci Pollut Res. 26:37242-37253. doi:10. 1007/s11356-019-06615-8

22. Venkatesan N, Punithavathi D, Arumugam V. 2000. Curcumin prevents Adriamycin nephrotoxicity in rats. $\mathrm{Br} J$ Pharmacol. 129:231-4. doi:10.1038/sj.bjp.0703067

23. Wang D, Yang Y, Zou X, Zheng Z, Zhang J. 2020. Curcumin ameliorates CKD-induced mitochondrial dysfunction and oxidative stress through inhibiting GSK-3 $\beta$ activity. J Nutr Biochem. 83:108404. doi:10.1016/j.jnutbio.2020.108404

24. Wei QY, Chen WF, Zhou B, Yang L, Liu ZL. 2006. Inhibition of lipid peroxidation and protein oxidation in rat liver mitochondria by curcumin and its analogues. Biochimica et Biophysica Acta.1760(1):70-7. doi:10.1016/j.bbagen.2005.09.008 Yang QQ, Farha AK, Kima G, Gula K, Ganb RY, Corke H. 2020. Antimicrobial and anticancer applications and related mechanisms of curcumin-mediated photodynamic treatments. Trends Food Sci Technol. 97:341-354. doi:10.1016/j.tifs.2020.01.023

25. Zhang F, Xu Z, Gao J, Xu B, Deng Y. 2008. In vitro effect of manganese chloride exposure on energy metabolism and oxidative damage of mitochondria isolated from rat brain. Environ Toxicol Pharmacol. 26(2):232-6. doi:10.1016/j.etap.2008.04.003

26. Zhao Y, Ye L, Liu H, Xia Q, Zhang Y, Yang X, Wang K. 2010. Vanadium compounds induced mitochondria permeability transition pore (PTP) opening related to oxidative stress. J Inorg Biochem. 104(4): 371-378. doi:10.1016/j.jinorgbio.2009.11.007

27. Zheng S, Yumei F, Chen, A. 2007. de novo synthesis of glutathione is a prerequisite for curcumin to inhibit HSC activation. Free Radic Biol Med. 43(3):444-53. doi:10.1016/j.freeradbiomed.2007.04.016 